\title{
Factores de riesgo psicosocial y molestias musculoesqueléticas en cajeros bancarios de una empresa bancaria en Lima - Perú
}

\author{
Psychosocial Risk Factors and Musculoskeletal \\ Discomfort Among Tellers at a Banking Company \\ in Lima, Peru
}

Richard Raitt Rodriguez-Rojas ${ }^{1}$ (1) 0000-0001-5083-6736

Carlos Manuel Escobar-Galindo² (1) 0000-0001-7263-9215

Paula Martha Veliz-Terry ${ }^{3}$ (1) 0000-0002-7332-2727

Roly Marino Jara-Espinoza ${ }^{4}$ (1) 0000-0003-2563-458X

`Escuela de Tecnología Médica, Universidad Nacional Mayor de San Marcos, Lima, Perú ${ }^{2}$ Human Factors Research Group, University of Nottingham, Nottingham, England, Union Kingdom.

${ }^{3}$ Escuela de Tecnología Médica, Universidad Nacional Mayor de San Marcos, Lima, Perú.

${ }^{4}$ Facultad de Medicina, Universidad Peruana Cayetano Heredia, Lima, Perú.

Fechas · Dates

Recibido: 2020.09.06 Aceptado: 2021.01.18 Publicado: 2021.04.15
Correspondencia · Corresponding Author

Richard Raitt Rodriguez-Rojas

richardraitt.rodriguez@unmsm.edu.pe 


\section{Resumen}

Objetivo: Determinar si la presencia de molestias musculoesqueléticas y el nivel de riesgo psicosocial se hallan relacionados en cajeros de una empresa bancaria en la ciudad de Lima.

Métodos: Diseño metodológico de tipo transversal y descriptivo. Se analizó una muestra de 234 trabajadores. Los instrumentos utilizados fueron el Cuestionario de Riesgos Psicosociales en el Trabajo SUSESO - ISTAS 21 VERSIÓN BREVE, una adaptación del Cuestionario Nórdico de Molestias Musculoesqueléticas y una ficha de datos sociodemográficos. Se aplicaron los instrumentos de valoración entre los meses septiembre y noviembre del año 2018. Se utilizó la prueba de Chi Cuadrado para analizar la relación entre las variables principales del estudio.

Resultados: Las dimensiones de factores psicosociales con más prevalencia de riesgo alto fueron "Exigencias psicológicas" (50.1\%) y "Doble presencia" (49.1\%). Las molestias musculoesqueléticas más frecuentes fueron las reportadas en el cuello (75.9\%) y en la zona dorsolumbar (75.0\%). Se observó relación entre el nivel de riesgo psicosocial en la dimensión "Compensaciones" y cantidad de regiones corporales afectadas por molestias musculoesqueléticas en los cajeros bancarios $(p<0.05)$.

Conclusiones: Existe relación entre el nivel de riesgo psicosocial en la dimensión de "Compensaciones" y la cantidad de regiones corporales con reporte de molestias musculoesqueléticas. Se recomienda propiciar factores psicosociales protectores para evitar las molestias musculoesqueléticas en los cajeros bancarios.

Palabras clave: Dolor musculoesquelético; Estrés Laboral; Satisfacción Laboral; Ergonomía; Cuenta Bancaria.

\section{Abstract}

Objective: We examined associations between psychosocial risk factors and musculoskeletal discomfort among tellers at a bank Lima, Peru.

Methods: This was a cross-sectional descriptive study of 234 workers. The instruments used were the Questionnaire of Psychosocial Risks at Work SUSESO - ISTAS 21 Short Version, an adaptation of the Nordic Musculoskeletal Discomfort Questionnaire and a sociodemographic data sheet. These two questionnaires were administered between September and November 2018. We analyzed associations between the two main study variables with chi-square tests.

Results: The most prevalent psychosocial factor dimensions were "psychological demands " (50.1\%) and "double presence" (49.1\%). The most frequent musculoskeletal discomfort were in the neck $(75.9 \%)$ and thoracolumbar (75\%) areas. We observed an association between the level of psychosocial risk in the "compensation" dimension and the number of body regions affected by musculoskeletal discomfort ( $p<0.05)$.

Conclusions: There is a relationship between the level of psychosocial risk in the "compensation" dimension and the number of body regions with reported musculoskeletal discomfort. We recommend implementing psychosocial risk factor preventive measures to avoid musculoskeletal discomfort in bank tellers.

Keywords: musculoskeletal pain; occupational stress; job satisfaction; ergonomics; banking personnel. 


\section{Introducción}

A raíz de la crisis económica mundial sumada a la modernización de las entidades bancarias, las personas han reducido su capacidad de ahorro e inversión, lo cual impulsa a los bancos a crear estrategias que modifiquen los patrones clásicos, apostando por nuevos esquemas de abordaje y redefiniendo la banca tradicional. En este contexto los trabajadores bancarios se encuentran expuestos a mayor presión de tiempo, mayor demanda de trabajo, conflicto de roles, intensificación del ritmo de trabajo y violencia por parte de clientes, lo que puede afectar su salud física y mental( ${ }^{(1)}$. Se ha identificado que las principales fuentes de estrés en este grupo incluyen la sobrecarga de trabajo y las malas condiciones de este, el desbalance entre el esfuerzo y la retribución, la ambigüedad y el estancamiento del rol, y el desequilibrio entre el trabajo y la vida privada. Además, están expuestos a horarios prolongados, actitudes gerenciales negativas, automatización e inseguridad laboral(2).

Dentro de este grupo, los cajeros bancarios, quienes manipulan dinero directamente, presentan mayor agotamiento emocional, estrés laboral y menor eficacia en comparación con los que trabajan en otras áreas, debido al componente interpersonal, la demanda comercial, la inestabilidad laboral, la exposición a violencia y la no conformidad salarial ${ }^{(3,4)}$. Por ser un puesto de atención al cliente, deben manifestar cortesía permanentemente, incluso si son agredidos verbalmente, por lo que los obliga a esconder sus emociones constantemente. Además, habitualmente el ritmo de trabajo es impuesto por la demanda de los clientes, de manera que deben hacer uso de sus capacidades cognitivas (memoria y concentración) a un alto nivel(5). La elevada carga de trabajo tanto a nivel cognitivo como emocional puede generar consecuencias negativas para la salud mental, como ansiedad y estrés. El Instituto Nacional de Seguridad y Salud Ocupacional (NIOSH) lo ubicó dentro de las 28 ocupaciones con niveles más altos de estrés(6).

Este desequilibrio entre la alta demanda del trabajo y bajo control del proceso, así como también un pobre sistema de retribución laboral, pueden ser factores cruciales para desencadenar estrés laboral y posibles respuestas emocionales. Estas condiciones negativas del trabajo relacionadas a la organización, las relaciones interpersonales y el tipo o diseño de tarea, son denominadas Factores de Riesgo Psicosocial (FRP) ${ }^{(7)}$, los cuales pueden agruparse en función de: condiciones laborales (pobre salario, pobre liderazgo, falta de sostenibilidad, deficientes condiciones de trabajo), organización del trabajo (pobre control del trabajo, alta demanda o carga de trabajo, pobre participación en decisiones, desequilibrio en las recompensas o pobre compensación, desequilibrio entre el trabajo y el hogar o doble presencia, horarios de trabajo inadecuados, trabajo por turnos), y relaciones (pobre apoyo social, acoso psicológico y sexual, conflictos interpersonales)(8).

Karasek explica en su "Modelo Demanda - Control", cómo los diferentes niveles de exigencias en el trabajo y de capacidad de control sobre los procesos pueden dar lugar diferentes escenarios, siendo uno de ellos el trabajo "altamente estresante" configurado por altas demandas y pobre control(9). Por otro lado, Siegrist en su "Modelo Desequilibrio Esfuerzo - Recompensa" indica que el estrés laboral surge 
a raíz del desajuste entre el esfuerzo llevado a cabo por el trabajador y la retribución (salarial, social, profesional) que recibe por parte de la empresa ${ }^{(10,11)}$.

Sin embargo, en el Perú, a pesar de la reciente legislación sobre Seguridad y Salud en el Trabajo (SST), la gestión de los FRP es muy limitada e, incluso, el actual reglamento de SST no contempla una definición clara ${ }^{(12)}$. Según el Centro de Prevención de Riesgos en el Trabajo del Perú, los FRP representan el 48.3\% de riesgos a los que están expuestos los trabajadores(13). Además, en un reciente informe, se establece que el 100\% de entidades bancarias en el Perú no poseen sistemas de compensaciones o incentivos a sus trabajadores ${ }^{(14)}$.

Por otro lado, los FRP no están solamente relacionados al estrés laboral o a variables de carácter psicológico, sino también al disconfort físico, lo cual se traduce en Molestias Musculoesqueléticas (MME); éstas son las percepciones subjetivas de dolor, fatiga y temblores provenientes de las diferentes regiones corporales. No implican necesariamente la presencia de un Trastorno Musculoesquelético (TME) específico y diagnosticado clínicamente, aunque pueden llegar a serlo; sin embargo, son de utilidad para tomar acciones preventivas, puesto que muchas MME devienen en $\operatorname{TME}^{(15,16)}$.

Las MME, por lo general, relacionan a aspectos de ergonomía física como sobrecarga física, movimientos repetitivos, esfuerzos o posturas forzadas; sin embargo, su origen también se genera bajo un contexto psicosocial determinado, influenciado por la capacidad de respuesta emocional del individuo y de sus expectativas ${ }^{(17)}$. La sensación de confort en el trabajo no solo se debe a la ausencia de disconfort, sino también a factores psicosociales protectores propios del trabajo ${ }^{(17)}$. En el Perú, las empresas bancarias han reportado un 57\% de TME, los cuales son atribuidos principalmente a factores de riesgo ergonómico ${ }^{(14)}$; sin embargo, es cierto que estas mismas empresas, también, poseen altos niveles de estrés laboral.

Ahora bien, la relación estadística entre la exposición a FRP y la prevalencia de TME aún no es del todo clara. Diversos estudios evidencian la asociación entre FRP y TME, relacionando la alta demanda de trabajo e insuficiente control, con reportes de TME en espalda(18); no obstante, la evidencia no es lo suficientemente sólida en comparación con los factores de riesgo ergonómico y físicos/ambientales $^{(19)}$.

Por otro lado, las actuales políticas de SST en las empresas peruanas separan ambos componentes (mental y físico) en programas totalmente distintos de modo que se obvian los aspectos relacionados al disconfort físico dentro de la gestión de factores de riesgo ergonómico ${ }^{(20,21)}$. Basado en estos antecedentes, surge la necesidad de analizar más a fondo la actividad laboral de los cajeros bancarios desde la perspectiva de los riesgos psicosociales para priorizar futuros planes de acción que faciliten un abordaje integral que pueda optimizar su desempeño laboral y alcanzar un adecuado bienestar físico y mental en los trabajadores ${ }^{(22)}$.

El presente estudio tiene como objetivo determinar si la presencia de MME y el nivel de riesgo psicosocial se hallan relacionados en cajeros bancarios de una empresa bancaria en Lima - Perú. 


\section{Métodos}

\section{Diseño del estudio}

El estudio fue de corte transversal, de tipo observacional y de nivel correlacional, aplicado entre los meses de septiembre y noviembre del año 2018, en cajeros bancarios de una empresa bancaria en Lima - Perú.

\section{Población y muestra}

Se consideró como población a todos los cajeros bancarios de las agencias de una empresa bancaria en Lima Metropolitana, constituyendo un total de 529 cajeros. Se incluyeron a los trabajadores que laboraban a tiempo completo, y se excluyeron a los que estuvieran en calidad de practicante.

Se calculó el tamaño de muestra considerando una proporción esperada de $51 \%{ }^{(19)}$, un nivel de confianza de $95 \%$ y un error de muestreo de $5 \%$, por lo que se obtuvo una muestra mínima de 223 participantes.

\section{Variables de estudio}

Las variables principales del estudio fueron: El "Nivel de Riesgo Psicosocial" y las "Molestias Musculoesqueléticas". El nivel de riesgo psicosocial para cada dimensión (o factor del instrumento) se estableció al realizar la suma simple de los puntajes obtenidos y ubicando el resultado en tres posibles niveles de riesgo: "bajo", "medio" y "alto"(11,23). Se consideraron las siguientes dimensiones: "Exigencias psicológicas en el trabajo", "Trabajo activo y desarrollo de habilidades", "Apoyo social en la empresa y calidad de liderazgo", "Compensaciones" y "Doble presencia".

Con respecto a las Molestias Musculoesqueléticas, se tomó en cuenta la presencia de molestias según las regiones anatómicas: cuello, hombros, codo - antebrazo, mano - muñeca, y dorsolumbar; debido a su alta prevalencia en estudios previos $^{(18)}$.

Se consideraron otras variables como el grupo etario (18-20 años, 21-36 años, 3755 años), el sexo, el tiempo en la empresa (menos de 1 año, 1 a 2 años, más de 2 años) y el tiempo diario trabajando en la computadora (menos de 8 horas diarias, más de 8 horas diarias).

\section{Instrumentos de recolección de datos}

Para obtener información sobre FRP, se usó el Cuestionario de evaluación de riesgos psicosociales en el trabajo SUSESO ISTAS - 21 VERSIÓN BREVE, una adaptación chilena del original(11,23). Para fines del presente estudio, el instrumento fue sometido a un juicio de expertos y a una prueba piloto de cuatro meses, estimando la confiabilidad mediante el cálculo del Alfa de Cronbach y considerándose aceptable $(a=0.8)^{(24)}$. 
Se usó una versión adaptada del Cuestionario Nórdico para el registro de las MME en las diferentes zonas del cuerpo ${ }^{(16)}$. Se organizó la información para que sea entendida con mayor facilidad y fue sometido a un juicio de expertos. Se estimó la confiabilidad mediante la fórmula de Kuder - Richardson (KR-20) considerándose aceptable $(a=0.7)^{(24)}$. Se contó con una ficha para la recolección de datos sociodemográficos.

Previo al estudio, se realizó una charla de sensibilización de 30 minutos de duración, que abordó los FRP en el trabajo y sus efectos en la salud. Posteriormente, se entregó el consentimiento informado para su aceptación; una vez aceptado, se les brindó el cuestionario SUSESO ISTAS - 21 VERSIÓN BREVE, el Cuestionario Nórdico de MME y la ficha de datos sociodemográficos. La recolección y análisis de datos se realizó en presencia de un experto en el campo de la ergonomía para garantizar la eficacia del procedimiento.

\section{Análisis estadísticos de datos}

Los resultados fueron procesados en el programa Microsoft Excel 2013, y en el software estadístico IBM SPSS Statistics versión 20. El análisis descriptivo de las variables categóricas se distribuyó en tablas y frecuencias. Se realizó un análisis bivariado para establecer las relaciones entre las variables principales, haciendo uso del estadígrafo Chi Cuadrado y la Prueba Exacta de Fisher según corresponda. Para todos los análisis estadísticos se utilizó un nivel de significación del 5\% $(p<0.05)$.

\section{Aspectos éticos}

Se consideraron los valores éticos universalmente aceptados como son la autonomía, justicia, beneficencia y no maleficencia. Se respetó la autonomía de los participantes informándoles las características del estudio a través del consentimiento informado. Todos gozaron de igualdad de trato, y se les aseguró la confidencialidad y anonimato de los resultados obtenidos. La participación en el estudio no supuso ningún peligro a la salud de los participantes, ni afectó negativamente su actividad laboral. El estudio fue aprobado éticamente por la Facultad de Medicina de la Universidad Nacional Mayor de San Marcos a través de resolución de decanato $N^{\circ}$ 2382-D-FM-2018. Se solicitó conformidad de la organización a través del área de SST.

\section{Resultados}

El total de participantes fue de 234, predominando los participantes del sexo femenino $(62.0 \%)$. Casi la totalidad de los trabajadores pertenecieron al rango de edad de entre 21 y 36 años (95.7\%) con más de 2 años en la empresa (44.0\%) y pasando más de 8 horas frente a la PC (72.2\%) (Tabla 1). 
Tabla 1: Datos Sociodemográficos e Información laboral.

\begin{tabular}{lcc}
\hline Datos Sociodemográficos & $\mathbf{n = 2 3 4}$ & Porcentaje (\%) \\
\hline Sexo & & 38.0 \\
\hline Masculino & 89 & 62.0 \\
\hline Femenino & 145 & \\
\hline Grupo etario & & 3.4 \\
\hline 18-20 años & 8 & 95.7 \\
\hline $21-36$ años & 224 & 0.9 \\
\hline 37-55 años & 2 &
\end{tabular}

Información Laboral

\begin{tabular}{lcc}
\hline Uso de computadora & & \\
\hline Menos 8 horas & 65 & 27.8 \\
\hline Más 8 horas & 169 & 72.2 \\
\hline Tiempo en empresa & & 32.1 \\
\hline Menos de 1 año & 75 & 23.9 \\
\hline De 1 a 2 años & 56 & 44.0 \\
\hline Más de 2 años & 103 & \\
\hline
\end{tabular}

Se identificó un elevado porcentaje de alto riesgo psicosocial en las dimensiones "Exigencias Psicológicas" (50.1\%) y "Doble Presencia" (49.1\%). La dimensión "Compensaciones" obtuvo un mayor riesgo en la categoría "moderado" (47.0\%), mientras que el "Trabajo activo" obtuvo 38.9\% de riesgo categorizado "moderado" y $38.8 \%$ de riesgo "alto". (Figura 1).

La prevalencia global de MME (al menos una región con reporte de molestia) fue de $91.4 \%$. El 72.4\% de los participantes reportaron MME en más de una región corporal; sobresaliendo la población femenina con el 68.5\% ( $p<0.05)$. Las mujeres presentaron mayor prevalencia de molestias en todas las regiones corporales, observándose diferencias significativas en la región del cuello y los hombros $(p<0.05)$ (Tabla 2). Las regiones donde se reportó mayor frecuencia fueron las molestias en zona cervical (75.9\%), dorsolumbar (75.0\%) y los hombros (42.2\%).

Más del 71\% de los participantes que reportaron MME en alguna de las regiones corporales trabajaban más de 8 horas diarias; sin embargo, no se halló relación entre la cantidad de horas diarias de trabajo y la presencia de molestias en alguna de las regiones corporales ( $p>0.05)$ (Figura 2). 


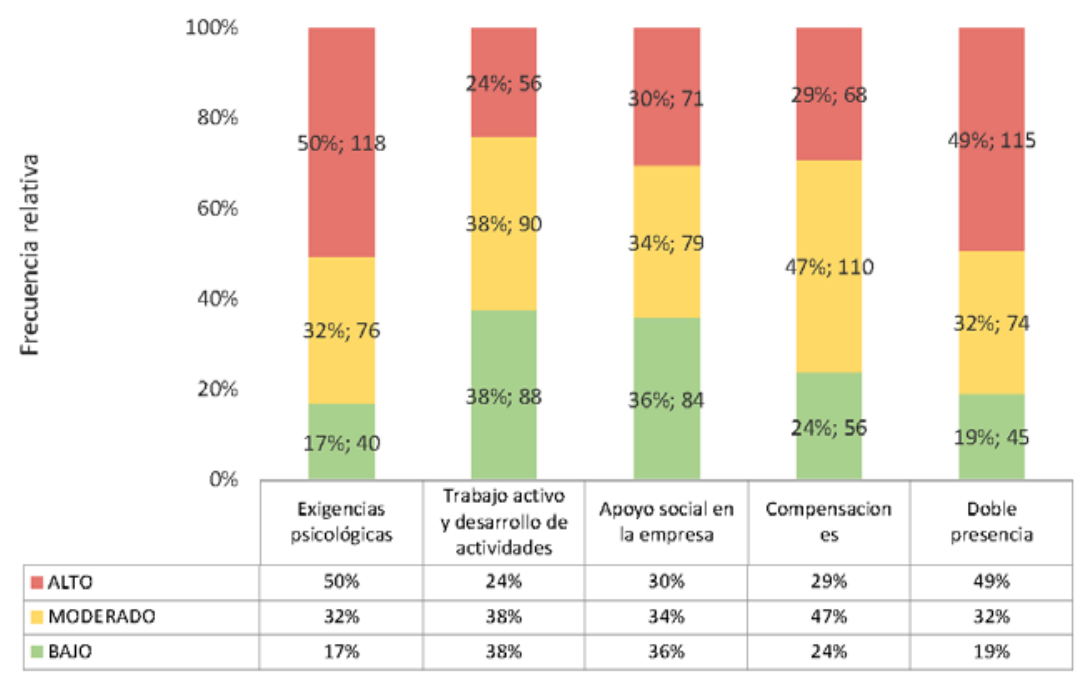

Factores de Riesgo Psicosocial

$=$ BAO $=$ MODERADO $=$ ALTO

Figura 1: Nivel de Riesgo Psicosocial.

Tabla 2: Prevalencia de molestias musculoesqueléticas por sexo.

\begin{tabular}{|c|c|c|c|c|c|c|}
\hline \multirow{3}{*}{$\begin{array}{l}\text { Molestias } \\
\text { Musculoesqueléticas } \\
\text { (MME) }\end{array}$} & & \multirow[b]{3}{*}{ valor $p^{a}$} & \multicolumn{4}{|c|}{ Sexo } \\
\hline & & & \multicolumn{2}{|c|}{ Masculino } & \multicolumn{2}{|c|}{ Femenino } \\
\hline & & & $n=89$ & $\begin{array}{l}\text { Prevalencia } \\
\quad(\%)\end{array}$ & $n=145$ & $\begin{array}{l}\text { Prevalencia } \\
\text { (\%) }\end{array}$ \\
\hline \multirow{3}{*}{$\begin{array}{l}\text { Cantidad de regiones } \\
\text { corporales con MME }\end{array}$} & Ninguna & \multirow{3}{*}{$0.001^{\star}$} & 15 & 16.9 & 7 & 4.8 \\
\hline & Una & & 21 & 23.6 & 23 & 15.9 \\
\hline & $\begin{array}{l}\text { Más de } \\
\text { una }\end{array}$ & & 53 & 59.6 & 115 & 79.3 \\
\hline \multirow{2}{*}{ MME en Cuello } & Sí & \multirow{2}{*}{$0.000^{*}$} & 51 & 57.3 & 125 & 86.2 \\
\hline & No & & 38 & 42.7 & 20 & 13.8 \\
\hline \multirow{2}{*}{ MME en Hombros } & Sí & \multirow{2}{*}{$0.005^{\star}$} & 27 & 30.3 & 71 & 49.0 \\
\hline & No & & 62 & 69.7 & 74 & 51.0 \\
\hline \multirow{2}{*}{ MME en Dorsolumbar } & Sí & \multirow{2}{*}{0.110} & 61 & 68.5 & 113 & 77.9 \\
\hline & No & & 28 & 31.5 & 32 & 22.1 \\
\hline \multirow{2}{*}{$\begin{array}{l}\text { MME en Codos- } \\
\text { Antebrazos }\end{array}$} & Sí & \multirow{2}{*}{0.398} & 15 & 16.9 & 31 & 21.4 \\
\hline & No & & 74 & 83.1 & 114 & 78.6 \\
\hline \multirow[t]{2}{*}{$\begin{array}{l}\text { MME en Manos- } \\
\text { Muñecas }\end{array}$} & Sí & \multirow[t]{2}{*}{0.604} & 28 & 31.5 & 41 & 28.3 \\
\hline & No & & 61 & 68.5 & 104 & 71.7 \\
\hline
\end{tabular}

a Prueba de Chi Cuadrado g.l. (2).

b Prueba de Chi Cuadrado g.l. (1).

* Asociación estadística $(p<0.05)$. 
Se identificó relación entre el nivel de riesgo psicosocial en la dimensión de "Compensaciones" y la cantidad de regiones corporales donde hubo reporte de MME $(p<0.05)$. Los participantes que presentaron más de una región afectada por MME tienden a poseer mayor riesgo psicosocial por "Compensaciones" $(z=3.3, p<0.01)$ (ver Tabla 3).

La mayor cantidad de participantes que registraron alto riesgo psicosocial en todas las dimensiones fueron mujeres, tenían más de dos años laborando en la empresa, y trabajaban más de 8 horas diarias. Se halló relación entre el sexo y el nivel de riesgo psicosocial en la dimensión de "Compensaciones" ( $p<0.05)$ (Tabla 4).

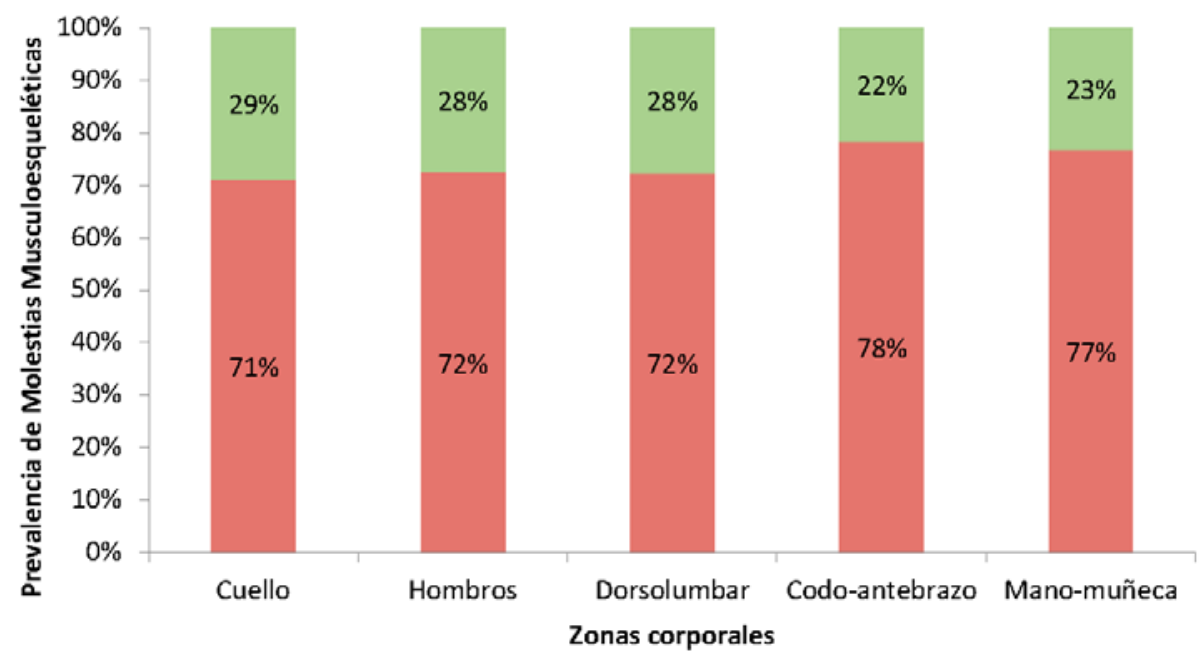

a $>8$ horas diarias usando computadora $\quad[<8$ horas diarias usando computadora

Figura 2: Prevalencia de molestias musculoesqueléticas en función del número de horas diarias de trabajo. 
Tabla 3: Prevalencia de molestias musculoesqueléticas en relación entre nivel de riesgo psicosocial.

\begin{tabular}{|c|c|c|c|c|c|c|c|}
\hline \multirow{3}{*}{$\begin{array}{l}\text { Factores de Riesgo } \\
\text { Psicosocial }^{\mathrm{a}}\end{array}$} & \multicolumn{7}{|c|}{ Cantidad de zonas afectadas } \\
\hline & \multirow[b]{2}{*}{ valor $\mathrm{p}^{\mathrm{b}}$} & \multicolumn{2}{|c|}{ Ninguna } & \multicolumn{2}{|r|}{ Una } & \multicolumn{2}{|c|}{ Más de una } \\
\hline & & $\mathrm{n}=22$ & $\begin{array}{l}\text { Porcentaje } \\
(\%)\end{array}$ & $\mathrm{n}=44$ & $\begin{array}{l}\text { Porcentaje } \\
(\%)\end{array}$ & $n=168$ & $\begin{array}{c}\text { Porcentaje } \\
(\%)\end{array}$ \\
\hline $\begin{array}{l}\text { Exigencias } \\
\text { Psicológicas }\end{array}$ & \multirow{4}{*}{0.131} & & & & & & \\
\hline Riesgo Alto & & 7 & 31.8 & 23 & 52.3 & 88 & 52.4 \\
\hline Riesgo Medio & & 11 & 50.0 & 17 & 38.6 & 48 & 28.6 \\
\hline Riesgo Bajo & & 4 & 18.2 & 4 & 9.1 & 32 & 19.0 \\
\hline \multicolumn{8}{|l|}{ Trabajo Activo } \\
\hline Riesgo Alto & \multirow{3}{*}{0.467} & 6 & 27.3 & 8 & 18.2 & 42 & 25.0 \\
\hline Riesgo Medio & & 11 & 50.0 & 19 & 43.2 & 60 & 35.7 \\
\hline Riesgo Bajo & & 5 & 22.7 & 17 & 38.6 & 66 & 39.3 \\
\hline \multicolumn{8}{|l|}{ Apoyo Social } \\
\hline Riesgo Alto & \multirow{3}{*}{0.288} & 5 & 22.7 & 13 & 29.5 & 53 & 31.5 \\
\hline Riesgo Medio & & 8 & 36.4 & 10 & 22.7 & 61 & 36.3 \\
\hline Riesgo Bajo & & 9 & 40.9 & 21 & 47.7 & 54 & 32.1 \\
\hline \multicolumn{8}{|l|}{ Compensaciones } \\
\hline Riesgo Alto & \multirow{3}{*}{$0.005^{\star z}$} & 3 & 13.6 & 9 & 20.5 & 56 & 33.3 \\
\hline Riesgo Medio & & 14 & 63.6 & 16 & 36.4 & 80 & 47.6 \\
\hline Riesgo Bajo & & 5 & 22.7 & 19 & 43.2 & 32 & 19.0 \\
\hline \multicolumn{8}{|l|}{ Doble Presencia } \\
\hline Riesgo Alto & \multirow{3}{*}{0.684} & 9 & 40.9 & 19 & 43.2 & 87 & 51.8 \\
\hline Riesgo Medio & & 8 & 36.4 & 14 & 31.8 & 52 & 31.0 \\
\hline Riesgo Bajo & & 5 & 22.7 & 11 & 25.0 & 29 & 17.3 \\
\hline
\end{tabular}

a De acuerdo con el Cuestionario de Evaluación de Riesgos Psicosociales en el Trabajo SUSESO - ISTAS 21.

b Prueba de Chi Cuadrado g.l. (4).

* Asociación estadística $(p<0.01)$ (residuo corregido $z=3.3$, riesgo alto - más de una molestia) 
Tabla 4: Datos Sociodemográficos e Información Laboral por Factores de riesgo psicosociales en nivel alto.

\begin{tabular}{|c|c|c|c|c|c|c|c|c|c|c|}
\hline \multicolumn{11}{|c|}{ Factores de Riesgo Psicosociala } \\
\hline \multirow{2}{*}{$\begin{array}{l}\text { Datos Socio- } \\
\text { demográficos } \\
\text { e Información } \\
\text { Laboral }\end{array}$} & \multicolumn{2}{|c|}{$\begin{array}{l}\text { Exigencias } \\
\text { Psicológicas }\end{array}$} & \multicolumn{2}{|c|}{$\begin{array}{l}\text { Trabajo } \\
\text { Activo }\end{array}$} & \multicolumn{2}{|c|}{ Apoyo Social } & \multicolumn{2}{|c|}{ Compensaciones } & \multicolumn{2}{|c|}{$\begin{array}{c}\text { Doble } \\
\text { Presencia }\end{array}$} \\
\hline & $n=118$ & $\%$ & $\mathrm{n}=56$ & $\%$ & $\mathrm{n}=71$ & $\%$ & $\mathrm{n}=68$ & $\%$ & $\mathrm{n}=68$ & $\%$ \\
\hline \multicolumn{11}{|l|}{ Sexo } \\
\hline Masculino & 41 & 34.8 & 20 & 35.7 & 24 & 33.8 & 17 & 25 & 40 & 34.8 \\
\hline Femenino & 77 & 65.3 & 36 & 64.3 & 47 & 66.2 & 51 & $75^{\star}$ & 75 & 65.2 \\
\hline \multicolumn{11}{|l|}{ Grupo etario } \\
\hline 18-20 años & 2 & 1.7 & 2 & 3.6 & 2 & 2.8 & 3 & 4.4 & 5 & 4.3 \\
\hline 21-36 años & 115 & 97.5 & 53 & 94.6 & 69 & 97.2 & 64 & 94.1 & 109 & 94.8 \\
\hline 37-55 años & 1 & 0.8 & 1 & 1.8 & 0 & 0 & 1 & 1.5 & 1 & 0.9 \\
\hline \multicolumn{11}{|l|}{$\begin{array}{l}\text { Tiempo diario } \\
\text { en } P C^{b}\end{array}$} \\
\hline menos 8 horas & 35 & 29.7 & 14 & 25 & 23 & 32.4 & 24 & 35.3 & 34 & 29.6 \\
\hline más 8 horas & 83 & 70.3 & 42 & 75 & 48 & 67.6 & 44 & 64.7 & 81 & 70.4 \\
\hline \multicolumn{11}{|l|}{$\begin{array}{l}\text { Tiempo en } \\
\text { empresa }\end{array}$} \\
\hline menos de 1 año & 34 & 28.8 & 20 & 35.7 & 20 & 28.2 & 21 & 30.9 & 34 & 29.6 \\
\hline 1 a 2 años & 30 & 25.4 & 11 & 19.6 & 20 & 28.2 & 19 & 27.9 & 33 & 28.7 \\
\hline más de 2 años & 54 & 45.8 & 25 & 44.6 & 31 & 43.7 & 28 & 41.2 & 48 & 41.7 \\
\hline
\end{tabular}

\section{Conclusiones}

Las "Exigencias psicológicas" fueron uno de los factores de riesgo psicosocial con mayor prevalencia de alto riesgo, los cuales hacen referencia a demandas cognitivas y emocionales que desbordan las capacidades y el control. La alta demanda diaria de operaciones bancarias ininterrumpida junto con el escaso nivel de control de los ritmos de trabajo pudo reflejarse en el elevado número de cajeros expuestos, lo cual podría explicarse bajo el modelo Demanda - Control(9). La actividad laboral de los cajeros es de naturaleza multitarea, lo que significa que las demandas cognitivas no solo provienen del procesamiento de depósitos y retiros, sino que también de la venta de productos financieros. Además, se encuentran sujetos a altas metas de producción y a constante evaluación por parte del cliente, lo cual condiciona el acceso a bonos por productividad. Los bonos configuran un alto ingreso salarial, por lo que procuran atender la mayor cantidad de clientes y realizar la mayor cantidad de operaciones para poder alcanzar la meta mensual. Se ha observado que el trabajo compuesto por actividades multitarea de alta complejidad cognitiva produce mayor excitabilidad mental y bajos rendimientos ${ }^{(25)}$. 
Otro componente importante en la carga de trabajo es la demanda emocional. Los errores tanto por exceso o por defecto en la contabilización de efectivo pueden suponer descuentos en sus salarios y posibles reclamos. Los cajeros tienen, además, un escaso control de las situaciones de trabajo teniendo que ofrecer al cliente un trato amable, cálido y asertivo indistintamente de la situación de turno, sea atendiendo a clientes demandantes o violentos; por esta razón, se considera que tienen alta carga emocional(3,26). En este escenario, se genera la elevada demanda de trabajo (cognitivo y emocional) y al mismo tiempo el escaso control sobre el mismo, donde se ve reflejado claramente el trabajo "altamente estresante" descrito por Karasek ${ }^{(9)}$.

Por otro lado, la prevalencia global de MME fue 91.4\%; porcentaje elevado en comparación con otros grupos ocupacionales como trabajadores forestales, agricultores y pesqueros (69\%), operarios de máquinas $(66 \%)$, y artesanos $(65 \%)^{(19)}$. Esto podría explicarse, por dos aspectos: a la alta carga estática sedente, movimientos repetitivos y sobreesfuerzos producto de las tareas administrativas ${ }^{(20)}$, y por el modelo de disconfort físico propuesto por De Looze ${ }^{(17)}$. En este modelo, el contexto laboral de los cajeros bancarios demanda interacción humana constante y atención al cliente, en comparación con otros grupos ocupacionales, lo cual incrementa las probabilidades de exposición a FRP, genera estrés y, posteriormente, un incremento de las $\mathrm{MME}^{(5)}$.

Se esperaba encontrar asociaciones especialmente entre los elevados niveles de riesgo de "Exigencias psicológicas" y las MME, debido a que las primeras podrían imponer a los trabajadores severa tensión muscular que podría manifestarse en disconfort en la región del cuello y espaldaa ${ }^{(18)}$; sin embargo, no se encontró evidencia directa. Esto podría deberse a factores protectores, tales como el trabajo activo o la implementación de la banca virtual que reduce de alguna manera la carga de clientes.

Se halló relación entre el nivel de riesgo psicosocial en la dimensión de "Compensaciones" y la cantidad de regiones corporales con MME, lo cual guarda similitud con lo encontrado por S. Zakerian y cols. ${ }^{(27)}$. El riesgo psicosocial en la dimensión "Compensaciones" puede ser explicado bajo el modelo "Desequilibrio Esfuerzo Recompensa" de Siegrist ${ }^{(10)}$. Probablemente, los cajeros bancarios perciben que la cantidad de horas invertidas y la demanda cognitivo - emocional que implica el trabajo no compensa lo obtenido en el plano salarial, profesional, laboral y social; más aún cuando casi la totalidad pertenece a un grupo etario laboralmente emergente con diversas oportunidades a disposición. Esta situación puede producir niveles de insatisfacción no solo para el trabajador, sino también para los clientes. Además, podría explicar incluso por qué el puesto de cajero bancario es uno de los que posee mayores niveles rotación en la empresa ${ }^{(2,28)}$. Diversos estudios en otros grupos ocupacionales encontraron asociación entre alta carga de trabajo y bajo apoyo social con los TME a nivel de cuello y hombros; mientras que la insatisfacción laboral y la alta carga de trabajo se asociaron con TME en espalda(18). Estos hallazgos concuerdan con aspectos relacionados a "Compensaciones" que puede ser interpretado como un factor compuesto por dos elementos: percep- 
ción de esfuerzo excesivo (expresado por la alta carga de trabajo) y percepción de recompensas insuficientes (expresado por la insatisfacción laboral y el pobre apoyo social). Diferentes modelos sostienen que el estrés puede jugar un rol mediador entre los FRP y los TME, actuando tanto a nivel fisiológico, perceptual como comportamental(29) y tornando más susceptible al tejido musculoesquelético al impacto biomecánico.

El estudio consideró las limitaciones del instrumento SUSESO - ISTAS VERSIÓN $B R E V E$, las cuales mostraron niveles de confiabilidad similares a las de otros estudios de la región ${ }^{(30)}$. Sin embargo, para reducir las limitaciones originales del instrumento se siguieron las recomendaciones de la American Educational Research Association (AERA) lo cual implicó: una previa sensibilización a los participantes sobre FRP, el ofrecimiento de un espacio adecuado y brindarles el tiempo suficiente para el desarrollo del cuestionario. Ambos son criterios muy importantes para la efectividad de la aplicación de herramientas de valoración del riesgo psicosocial $^{(31)}$. Para futuras investigaciones, se sugiere utilizar la versión completa del cuestionario SUSESO - ISTAS 21 a fin de profundizar en aspectos específicos, complementando con entrevistas y observaciones en campo de tal forma que se pueda ahondar más en los aspectos vinculantes hallados en la investigación ${ }^{(30)}$. El estudio tuvo principalmente población medianamente joven, puesto que los cajeros bancarios conforman la primera línea de ascenso en el banco representando la realidad de este grupo ocupacional(28).

Se sugiere contemplar la gestión de los FRP como elemento indispensable no solo para reducir las respuestas de estrés del trabajador, sino también para reducir las $\mathrm{MME}$; idea que podría comenzar desde el desarrollo de un programa de compensaciones a los trabajadores. Este programa podría informar sobre los actuales beneficios disponibles, los cuales no son muchas veces visibles por los trabajadores; así como también estableciendo incentivos cuando se logren las metas propuestas a modo de retroalimentación. Estos programas deberían considerar las expectativas y emociones de los cajeros, los cuales son requisitos indispensables para alcanzar niveles de confort que permitan lograr satisfacción y bienestar del trabajador ${ }^{(17)}$

Finalmente, el trabajo ofrece un primer bosquejo de la influencia de los FRP en las MME de los cajeros bancarios, pero, a su vez, propone trabajar de manera integral sugiriendo estudios complementarios que aporten a la comprensión de la dinámica del sistema laboral, de manera que puedan brindarse recomendaciones respecto a mecanismos regulatorios de la actividad que favorezcan el bienestar, la salud, y la optimización del desempeño en el trabajo.

\section{Agradecimientos}

Al Dr. Álvaro Whittembury Vlásica por asesoría estadística y la Mg. Mirtha Sánchez Casas por asesoría temática.

\section{Financiación}

Autofinanciado.

\section{Conflicto de intereses}

Los autores declaran no tener conflictos de interés con la publicación de este artículo. 


\section{Bibliografía}

1. Giorgi G, Arcangeli G, Perminiene M, Lorini C, Ariza-Montes A, Fiz-Perez J, et al. Work-Related Stress in the Banking Sector: A Review of Incidence, Correlated Factors, and Major Consequences. Frontiers in psychology. 2017;8:2166.

2. Stańczak A, Mościcka-Teske A, Merecz-Kot D. Psychosocial risks and the job activity of banking sector employees. Med Pr. 2014;65(4):507-19.

3. Amigo I, Asensio E, Menéndez I. Working in direct contact with the public as a predictor of burnout in the banking sector. Psicothema. 2014;(26.2):222-6.

4. Mutsvunguma $P$, Gwandure $C$. The psychological well-being of employees who handle cash in a bank in inner city Johannesburg. Psychology, Health \& Medicine. 2011;16(4):430-6.

5. Michailidis M, Georgiou Y. Employee occupational stress in banking. Work. 2003;24:123-37.

6. Bureau of Labor Statistics, U.S. Department of Labor. Occupational Outlook Handbook, Cashiers [Internet]. [citado 12 Feb 2021]; Disponible en: https://www. bls.gov/ooh/sales/cashiers.htm

7. Gil-Monte PR. Riesgos psicosociales en el trabajo y salud ocupacional. Rev Peru Med Exp Salud Publica. 2012;29(2):237-41.

8. Kortum E, Leka S, Cox T. Psychosocial risks and work-related stress in developing countries: Health impact, priorities, barriers and solutions. Int J Occup Med Environ Health. 2010;23(3):225-38.

9. Karasek RA, Theorell T, Schwartz JE, Schnall PL, Pieper CF, Michela JL. Job characteristics in relation to the prevalence of myocardial infarction in the US Health Examination Survey (HES) and the Health and Nutrition Examination Survey (HANES). Am J Public Health. 1988;78(8):910-8.

10. Siegrist J. Adverse Health Effects of High-Effort/Low-Reward Conditions. J Occup Health Psychol. 1996;1(1):27-41.

11. Candia M, Pérez-Franco JM, González D. Manual del Método del Cuestionario SUSESO/ISTAS21 Versiones completa y breve. [Internet]. 2016. [citado 12 Feb 2021]. Disponible en: https://www.suseso.cl/613/articles-481095_archivo_03.pdf

12. Ministerio del Trabajo y Promoción del Empleo. Reglamento de la Ley $\mathrm{N}^{\circ}$ 29783, Ley de Seguridad y Salud en el Trabajo. DS Nº 005-2012 TR 2016.

13. Instituto Salud y Trabajo (ISAT). Diagnóstico Situacional en Seguridad y Salud en el Trabajo en el Perú [Internet]. Perú: Instituto Salud y Trabajo (ISAT); 2011. [citado 12 Feb 2021] Disponible en: https://www.isat.org.pe/publicaciones/item/78-ultimas-publicaciones

14. Marsh R. Realidad en la gestión de Seguridad y Salud en el Trabajo [Internet]. 2019. [citado 12 Feb 2021]. Disponible en: https://www.marsh.com/content/dam/ 
marsh/Documents/PDF/LAC/peru/mc-realidad-de-seguridad-y-salud-en-el-trabajo.pdf

15. Punnett L, Wegman DH. Work-related musculoskeletal disorders: the epidemiologic evidence and the debate. J Electromyogr Kinesiol. 2004; 14(1):13-23.

16. Martinez MM, Muñoz RA. Validation of the Nordic standardized questionnaire of musculoskeletal symptoms for the Chilean working population, including a pain scale. Revista de Salud Pública. 2017; 21(2), 43-53

17. De Looze MP, Kuijt-Evers LFM, Van Dieën J. Sitting comfort and discomfort and the relationships with objective measures. Ergonomics. 2003;46(10):985-97.

18. National Institute for Occupational Safety and Health. Musculoskeletal disorders and workplace factors. A critical review of epidemiologic evidence for work-related musculoskeletal disorders of the neck, upper extremity, and low back. [Internet]. U.S. Department of Health and Human Services, Public Health Service, Centers for Disease Control and Prevention, National Institute for Occupational Safety and Health; 1997 [citado 4 jun 2020]. Disponible en: https://www.cdc.gov/ niosh/docs/97-141/

19. European Agency for Safety and Health at Work. Work-related musculoskeletal disorders: prevalence, costs and demographics in the EU [Internet]. Luxembourg: Publications Office of the European Union; 2019. [citado 4 jun 2020]. Disponible en: https://osha.europa.eu/en/publications/msds-facts-and-figures-overview-prevalence-costs-and-demographics-msds-europe/view

20. Cáceres-Muñoz VS, Magallanes-Meneses AA, Torres-Coronel D, Copara-Moreno P, Escobar-Galindo M, Mayta-Tristán P. Effect of rest pauses combined with information leaflets on the decrease in musculoskeletal pain in administrative workers. Rev Peru Med Exp Salud Publica. 2017;34(4):611-8.

21. Palomar MDRL, García Cueva SA, Pando Moreno M. Factores de Riesgo Psicosocial y Burnout en Población Económicamente Activa de Lima, Perú. Ciencia \& trabajo. 2014;16(51):164-9.

22. Gimeno D. Los factores de riesgo laboral de naturaleza psicosocial y su prevención. Arch Prev Riesgos Labor. 2004;7(3):119-20.

23. Alvarado R, Pérez-Franco J, Saavedra N, Fuentealba C, Alarcón A, Marchetti N, et al. Validación de un cuestionario para evaluar riesgos psicosociales en el ambiente laboral en Chile. Rev Med Chil.;140(9):1154-63.

24. Oviedo HC, Campo-Arias A. Metodología de investigación y lectura crítica de estudios. Rev Colomb Psiquiatr. 2005;(4):9:136-143.

25. Adler RF, Benbunan-Fich R. The Effects of Task Difficulty and Multitasking on Performance. Interact Comput. 2015;27(4):430-9.

26. Sanda M-A, Mawuena E. Understanding the Effect of Emotional Exhaustion on Tellers' Job Satisfaction in Teller-Task Activity in Ghanaian Retail Banks. En: Kantola JI, Nazir S, Barath T, editores. Advances in Human Factors, Business Ma- 
nagement and Society [Internet]. Cham: Springer International Publishing; 2019. p. 461-72.

27. Zakerian S, Subramaniam I. Examining the Relationship between Psychosocial Work Factors and Musculoskeletal Discomfort among Computer Users in Malaysia. Iranian journal of public health. 2011;40:72-79.

28. Babakus E, Yavas U, Ashill NJ. Service worker burnout and turnover intentions: Roles of person-job fit, servant leadership, and customer orientation. Services Marketing Quarterly. 2011;32(1):17-31.

29. Eatough EM, Way JD, Chang C-H. Understanding the link between psychosocial work stressors and work-related musculoskeletal complaints. Appl Ergon. 2012;43(3):554-63.

30. Mendoza-Llanos R, Moyano-Díaz E. Hacia la validación del SUSESO ISTAS 21 versión breve en trabajadores de hospitales públicos. Terapia Psicológica. 2019;37(1):15-23.

31. American Educational Research Association (AERA); American Psychological, American Psychological Association (APA), National Council on Measurement in Education (NCME). Standards for educational and psychological testing. [Internet]. 2014. [citado 4 jun 2020]. Disponible en: https://eric.ed.gov/?id=ED565876. 